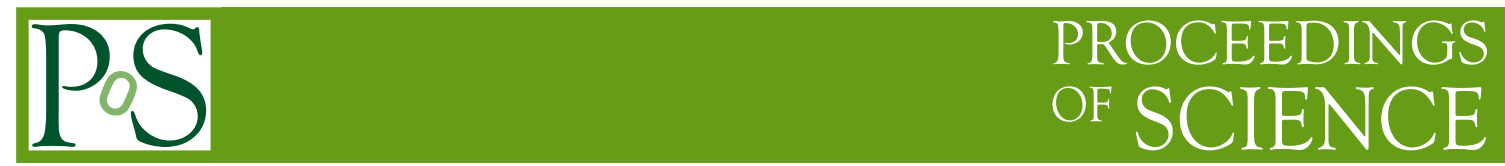

\title{
Higgs portal dark matter and the LHC
}

\section{Oleg Lebedev*}

DESY, Notkestrasse 85, Hamburg 22609, Germany

E-mail: lebedev@mail.desy.de

We discuss implications of the $125 \mathrm{GeV}$ Higgs signal at the LHC for Higgs portal dark matter $(\mathrm{DM})$ of different spins. We find that light scalar and vector $\mathrm{DM}$, i.e. with masses below $\approx 60$ $\mathrm{GeV}$, is strongly disfavored by the invisible Higgs decay. The fermionic DM is practically ruled out by a combination of the XENON100 and WMAP data.

Proceedings of the Corfu Summer Institute 2012

September 8-27, 2012

Corfu, Greece

* Speaker. 
The ATLAS and CMS collaborations have recently observed a resonance whose properties match closely those expected of the Standard Model (SM) Higgs boson [1]. In this contribution, we study implications of this result for Higgs-portal models of dark matter (DM). The Higgs sector of the SM enjoys a special status since it allows for a direct coupling to the hidden sector that is renormalizable. Hence, determination of the properties of the Higgs boson would allow us to gain information about the hidden world. The latter is particularly important in the context of dark matter since hidden sector particles can be stable and couple very weakly to the SM sector, thereby offering a viable dark matter candidate [2]. In principle, the Higgs boson could decay into light DM particles which escape detection. However, given the fact that the ATLAS and CMS signal is close to what one expects for a Standard Model-like Higgs particle, there is little room for invisible decays. In what follows, we will assume that $10 \%$ is the upper bound on the invisible Higgs decay branching ratio, although values up to $20 \%$ will not significantly change our conclusions.

Stability of dark matter is ensured by a $Z_{2}$ symmetry, which can be a consequence of gauge symmetry in the hidden sector [3]. The relevant $Z_{2}$-invariant terms in the Lagrangians are

$$
\begin{aligned}
& \Delta \mathscr{L}_{S}=-\frac{1}{2} m_{S}^{2} S^{2}-\frac{1}{4} \lambda_{S} S^{4}-\frac{1}{4} \lambda_{h S S} H^{\dagger} H S^{2}, \\
& \Delta \mathscr{L}_{V}=\frac{1}{2} m_{V}^{2} V_{\mu} V^{\mu}+\frac{1}{4} \lambda_{V}\left(V_{\mu} V^{\mu}\right)^{2}+\frac{1}{4} \lambda_{h V V} H^{\dagger} H V_{\mu} V^{\mu}, \\
& \Delta \mathscr{L}_{f}=-\frac{1}{2} m_{f} \bar{\chi} \chi-\frac{1}{4} \frac{\lambda_{h f f}}{\Lambda} H^{\dagger} H \bar{\chi} \chi .
\end{aligned}
$$

Here $S$ is a real scalar, $V_{\mu}$ is a vector and $\chi$ is a Majorana fermion. Although in the fermionic case the Higgs-DM coupling is not renormalizable, we still include it for completeness. The selfinteraction terms $S^{4}$ in the scalar case and the $\left(V_{\mu} V^{\mu}\right)^{2}$ term in the vector case are not essential for our discussion and we will ignore them. After electroweak symmetry breaking, the neutral component of the doublet field $H$ is shifted to $H^{0} \rightarrow v+h / \sqrt{2}$ with $v=174 \mathrm{GeV}$ and the physical masses of the DM particles will be given by

$$
\begin{aligned}
& M_{S}^{2}=m_{S}^{2}+\frac{1}{2} \lambda_{h S S} v^{2}, \\
& M_{V}^{2}=m_{V}^{2}+\frac{1}{2} \lambda_{h V V} v^{2}, \\
& M_{f}=m_{f}+\frac{1}{2} \frac{\lambda_{h f f}}{\Lambda} v^{2} .
\end{aligned}
$$

The relic abundance of the DM particles is obtained through the $s$-channel annihilation via the exchange of the Higgs boson. For instance, the annihilation cross section into light fermions of mass $m_{\text {ferm }}$ is given by

$$
\begin{aligned}
& \left\langle\sigma_{\text {ferm }}^{S} v_{r}\right\rangle=\frac{\lambda_{h S S}^{2} m_{\text {ferm }}^{2}}{16 \pi} \frac{1}{\left(4 M_{S}^{2}-m_{h}^{2}\right)^{2}}, \\
& \left\langle\sigma_{\text {ferm }}^{V} v_{r}\right\rangle=\frac{\lambda_{h V V}^{2} m_{\text {ferm }}^{2}}{48 \pi} \frac{1}{\left(4 M_{V}^{2}-m_{h}^{2}\right)^{2}}, \\
& \left\langle\sigma_{\text {ferm }}^{f} v_{r}\right\rangle=\frac{\lambda_{h f f}^{2} m_{\text {ferm }}^{2}}{32 \pi} \frac{M_{f}^{2}}{\Lambda^{2}} \frac{v_{r}^{2}}{\left(4 M_{f}^{2}-m_{h}^{2}\right)^{2}},
\end{aligned}
$$


where $v_{r}$ is the DM relative velocity. We should note that in our numerical analysis, we take into account the full set of relevant diagrams and channels, and we have adapted the program micrOMEGAs to calculate the relic DM density.

The properties of the dark matter particles can be studied in direct detection experiments. The DM interacts elastically with nuclei through the Higgs boson exchange. The resulting nuclear recoil is then interpreted in terms of the DM mass and DM-nucleon cross section. The spin-independent DM-nucleon interaction can be expressed as [4]

$$
\begin{aligned}
\sigma_{S-N}^{S I} & =\frac{\lambda_{h S S}^{2}}{16 \pi m_{h}^{4}} \frac{m_{N}^{4} f_{N}^{2}}{\left(M_{S}+m_{N}\right)^{2}}, \\
\sigma_{V-N}^{S I} & =\frac{\lambda_{h V V}^{2}}{16 \pi m_{h}^{4}} \frac{m_{N}^{4} f_{N}^{2}}{\left(M_{V}+m_{N}\right)^{2}}, \\
\sigma_{f-N}^{S I} & =\frac{\lambda_{h f f}^{2}}{4 \pi \Lambda^{2} m_{h}^{4}} \frac{m_{N}^{4} M_{f}^{2} f_{N}^{2}}{\left(M_{f}+m_{N}\right)^{2}},
\end{aligned}
$$

where $m_{N}$ is the nucleon mass and $f_{N}$ parameterizes the Higgs-nucleon coupling.

If the DM particles are light enough, $M_{\mathrm{DM}} \leq \frac{1}{2} m_{h}$, they will appear as invisible decay products of the Higgs boson. For the various cases, the Higgs partial decay widths into invisible DM particles are given by

$$
\begin{aligned}
\Gamma_{h \rightarrow S S}^{\mathrm{inv}} & =\frac{\lambda_{h S S}^{2} v^{2} \beta_{S}}{64 \pi m_{h}}, \\
\Gamma_{h \rightarrow V V}^{\mathrm{inv}} & =\frac{\lambda_{h V V}^{2} v^{2} m_{h}^{3} \beta_{V}}{256 \pi M_{V}^{4}}\left(1-4 \frac{M_{V}^{2}}{m_{h}^{2}}+12 \frac{M_{V}^{4}}{m_{h}^{4}}\right), \\
\Gamma_{h \rightarrow \chi \chi}^{\mathrm{inv}} & =\frac{\lambda_{h f f}^{2} v^{2} m_{h} \beta_{f}^{3}}{32 \pi \Lambda^{2}},
\end{aligned}
$$

where $\beta_{X}=\sqrt{1-4 M_{X}^{2} / m_{h}^{2}}$. We have adapted the program HDECAY which calculates all Higgs decay widths and branching ratios to include invisible decays.

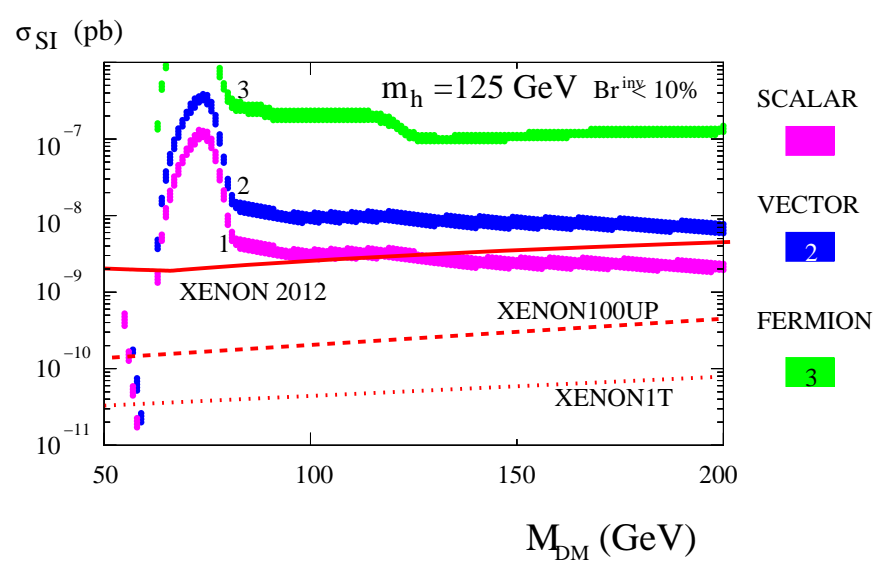

Figure 1: Spin independent DM-nucleon cross section versus DM mass. The upper band (3) corresponds to fermion DM, the middle one (2) to vector DM and the lower one (1) to scalar DM. The solid, dashed and dotted lines represent XENON100, XENON100 upgrade and XENON1T sensitivities, respectively. 
Our main results [5] are presented in Fig. 1 which displays predictions for the spin-independent DM-nucleon cross section $\sigma_{\mathrm{SI}}$ (based on the lattice $f_{N}$ ) subject to the WMAP and $\mathrm{BR}^{\text {inv }}<10 \%$ bounds. The upper band corresponds to the fermion Higgs-portal DM and is excluded by XENON100. On the other hand, scalar and vector DM are both allowed for a wide range of masses. Apart from a very small region around $\frac{1}{2} m_{h}$, this parameter space will be probed by XENON100-upgrade and XENON1T. The typical value for the scalar $\sigma_{\mathrm{SI}}$ is a few times $10^{-9} \mathrm{pb}$, whereas $\sigma_{\mathrm{SI}}$ for vectors is larger by a factor of 3 which accounts for the number of degrees of freedom.

We conclude that the entire class of Higgs-portal DM models will be probed by the XENON100upgrade and XENON1T direct detection experiments, which will also be able to discriminate between the vector and scalar cases. The fermion DM is essentially ruled out by the current data, most notably by XENON100. Furthermore, we find that light Higgs-portal DM $M_{\mathrm{DM}}<60 \mathrm{GeV}$ is excluded independently of its nature since it predicts a large invisible Higgs decay branching ratio, which is incompatible with the production of an SM-like Higgs boson at the LHC.

\section{References}

[1] F. Gianotti (for ATLAS) and G. Tonelli (for CMS), talks given at the CERN seminar on update on the Standard Model Higgs searches, CERN, 13/12/2011.

[2] V. Silveira, A. Zee, Phys. Lett. B161, 136 (1985).

[3] O. Lebedev, H. M. Lee and Y. Mambrini, Phys. Lett. B 707, 570 (2012).

[4] S. Kanemura, S. Matsumoto, T. Nabeshima, N. Okada, Phys. Rev. D82 (2010) 055026.

[5] A. Djouadi, O. Lebedev, Y. Mambrini and J. Quevillon, Phys. Lett. B 709, 65 (2012). 